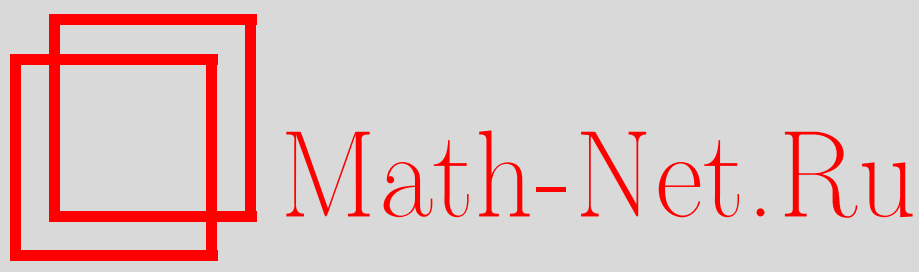

Г. Г. Магарил-Ильяев, К. Ю. Осипенко, Оптимальное восстановление функций и их производных по коэффициентам Фурье, заданным с погрешностью, Матем. сб., 2002, том 193, номер 3, 79-100

DOI: https://doi.org/10.4213/sm637

Использование Общероссийского математического портала Math-Net.Ru подразумевает, что вы прочитали и согласны с пользовательским соглашением

http://www . mathnet.ru/rus/agreement

Параметры загрузки:

IP: 3.82 .47 .9

26 апреля 2023 г., 05:18:29 


\title{
Оптимальное восстановление функций и их производных по коэффициентам Фурье, заданным с погрешностью
}

\begin{abstract}
В работе рассматриваются задачи оптимального восстановления функций и их производных по приближенным значениям коэффициентов Фурье. Приводятся явные выражения оптималњных методов восстановления для классов гладких и аналитических функций, определенных на различных компактных многообразиях.

Библиографояи: 9 названий.
\end{abstract}

\section{§1. Постановка задачи}

Начнем с общей постановки задачи об оптимальном восстановлении. Пусть $X-$ векторное пространство, $Z$-нормированное пространство и $T: X \rightarrow Z$-линейный оператор. Требуется восстановить значения $T$ на множестве (классе) $W \subset X$ по некоторой информации об элементах из этого класса. Точнее говоря, про каждьй элемент $x \in W$ мы располагаем информацией $I(x)$, где $I$ - некоторое отображение (называемое информационным) из $W$ в векторное пространство $Y$. Информация об элементах из $W$ может быть задана неточно, и поэтому $I$, вообше говоря, многозначное отображение.

В качестве метода восстановления допускается любое отображение $\varphi: Y \rightarrow Z$. Погрешностью этого метода называется величина

$$
e(T, W, I, \varphi)=\sup _{\substack{x \in W \\ y \in I(x)}}\|T x-\varphi(y)\|_{Z}
$$

Величина

$$
E(T, W, I)=\inf _{\varphi: Y \rightarrow Z} e(T, W, I, \varphi)
$$

носит название погрешности оптимального восстановления, а метод, на котором достигается нижняя грань, называется оптимальным методом восстановления (оператора $T$ на классе $W$ по информации $I$ ).

В работе изучается ситуация, когда $X$ - некоторое подпространство функций в $L_{2}(M)$, где $M$ - компактное многообразие (например, окружность, $d$-мерная сфера, круг в комплексной плоскости), $W \subset X$ - класс функций такой, что его частными случаями являются различные классы гладких и аналитических функций (например, классы Соболева, Харди-Соболева, Бергмана-Соболева), $T: X \rightarrow M-$

Работа выполнена при финансовой поддержке Российского фонда фундаменталњных исследований (гранты № № 99-01-01181 и 00-15-96109), а также при поддержке U.S.CRDFR.F.Ministry of Education Award VZ-010-0.

$$
\text { (C) Г. Г. МАГАРИЛ-ИЛЬЯЕв, К. Ю. ОСИПЕнкО }
$$


оператор мультипликаторного типа, частным случаем которого является оператор дифференцирования, и информация об $x(\cdot) \in W$ заключается в том, что нам известны с некоторой погрешностью (в той или иной метрике) все или конечное число коэффициентов Фурье функции $x(\cdot)$.

Задача восстановления линейных операторов в гильбертовых пространствах, когда $I$ - линейный оператор (т.е. информация задана точно), изучалась в работе [1]. В случае, когда информационное отображение $I$ со значениями в гильбертовом пространстве есть сумма линейного оператора и шара некоторого радиуса (задающего погрешность), соответствующая задача восстановления рассматривалась в работе [2] (см. также [3]-[5]). В [2], в частности, доказано, что среди оптимальных методов восстановления имеется линейный, и предложен некий способ его нахождения. Мы не пользуемся этим результатом. Наш подход основан на стандартных принципах выпуклой оптимизации, являющихся естественным инструментом решения подобного рода задач (о решении задач восстановления линейных функционалов с общих позиций теории экстремума см. [6]-[8]). Такой подход позволяет получить явные выражения для оптимальных методов восстановления и в тех случаях, когда погрешность информационного оператора задается в равномерной метрике.

В настоящей работе сначала подробно рассматриваются задачи восстановления для классов функций, заданных на окружности. Мы доказываем результаты достаточно общего характера и извлекаем из них следствия для различных классов гладких и аналитических функций. Незначительная модификация этих результатов позволяет получать аналогичные утверждения для классов функций на других многообразиях, что иллюстрируется на классах функций, заданных на $d$-мерной сфере и на единичном круге комплексной плоскости. Список подобных примеров может быть продолжен.

Перейдем к точному описанию класса $W$, оператора $T$ и информационных отображений $I$ в случае, когда $M=\mathbb{T}$. Пусть $x(\cdot)$ принадлежит пространству $L_{2}(\mathbb{T})$ с нормой

$$
\|x(\cdot)\|_{L_{2}(\mathbb{T})}=\left(\frac{1}{2 \pi} \int_{\mathbb{T}}|x(t)|^{2} d t\right)^{1 / 2}
$$

и

$$
x_{j}=\frac{1}{2 \pi} \int_{\mathbb{T}} x(t) e^{-i j t} d t, \quad j \in \mathbb{Z},
$$

- коэффициенты Фурье $x(\cdot)$. Пусть, далее, $\nu=\left\{\nu_{j}\right\}_{j \in \mathbb{Z}}-$ последовательность неотрицательных чисел. Сопоставим $\nu$ следующее подпространство в $L_{2}(\mathbb{T})$

$$
X=X^{\nu}(\mathbb{T})=\left\{x(\cdot) \in L_{2}(\mathbb{T}): \sum_{j \in \mathbb{Z}} \nu_{j}\left|x_{j}\right|^{2}<\infty\right\}
$$

и соответствующий класс

$$
W=W^{\nu}(\mathbb{T})=\left\{x(\cdot) \in X: \sum_{j \in \mathbb{Z}} \nu_{j}\left|x_{j}\right|^{2} \leqslant 1\right\} .
$$


Приведем примеры классов такого типа. Прежде всего, это соболевский класс $W_{2}^{r}(\mathbb{T})$, состоящий из $2 \pi$-периодических функций $x(\cdot)$, у которых $(r-1)$-я производная абсолютно непрерывна и $\left\|x^{(r)}(\cdot)\right\|_{L_{2}(\mathbb{T})} \leqslant 1$. Положив

$$
X=\left\{x(\cdot) \in L_{2}(\mathbb{T}): \sum_{j \in \mathbb{Z}} j^{2 r}\left|x_{j}\right|^{2}<\infty\right\}
$$

по теореме Планшереля получим эквивалентное определение соболевского класса:

$$
W_{2}^{r}(\mathbb{T})=\left\{x(\cdot) \in X: \sum_{j \in \mathbb{Z}} j^{2 r}\left|x_{j}\right|^{2} \leqslant 1\right\}
$$

Таким образом, $W_{2}^{r}(\mathbb{T})=W^{\nu}(\mathbb{T})$, где $\nu=\left\{j^{2 r}\right\}_{j \in \mathbb{Z}}$.

Обозначим через $\mathscr{H}_{2}^{\beta}(\mathbb{T})$ пространство Харди $2 \pi$-периодических функций $x(\cdot)$, аналитически продолжаемых в полосу $S_{\beta}=\{z \in \mathbb{C}:|\operatorname{Im} z|<\beta\}$ и удовлетворяющих условию

$$
\|x(\cdot)\|_{\mathscr{H}_{2}^{\beta}(\mathbb{T})}=\sup _{0<\rho<\beta}\left(\frac{1}{4 \pi} \int_{\mathbb{T}}\left(|x(t+i \rho)|^{2}+|x(t-i \rho)|^{2}\right) d t\right)^{1 / 2}<\infty .
$$

Пространством Бергмана $\mathscr{A}_{2}^{\beta}(\mathbb{T})$ называется множество $2 \pi$-периодических функций $x(\cdot)$, аналитически продолжаемых в полосу $S_{\beta}$ и удовлетворяющих услоВию

$$
\|x(\cdot)\|_{\mathscr{A}_{2}^{\beta}(\mathbb{T})}=\left(\frac{1}{4 \pi \beta} \int_{\mathbb{T}} d t \int_{-\beta}^{\beta}|x(t+i \rho)|^{2} d \rho\right)^{1 / 2}<\infty .
$$

Классы Харди-Соболева $H_{2}^{r, \beta}(\mathbb{T})$ и Бергмана-Соболева $A_{2}^{r, \beta}(\mathbb{T})$ определяются как множества $2 \pi$-периодических функций $x(\cdot)$, аналитически продолжаемых в полосу $S_{\beta}$ и удовлетворяющих условию $\left\|x^{(r)}(\cdot)\right\|_{\mathscr{H}_{2}^{\beta}(\mathbb{T})} \leqslant 1$ и $\left\|x^{(r)}(\cdot)\right\|_{\mathscr{A}_{2}^{\beta}(\mathbb{T})} \leqslant 1$ соответственно.

Функции из пространства Харди $\mathscr{H}_{2}^{\beta}(\mathbb{T})$ имеют почти всюду граничные значения, а само пространство $\mathscr{H}_{2}^{\beta}(\mathbb{T})$ является гильбертовым со скалярным произведением

$$
(x(\cdot), y(\cdot))_{\mathscr{H}_{2}^{\beta}(\mathbb{T})}=\frac{1}{4 \pi} \int_{\mathbb{T}}(x(t+i \beta) \overline{y(t+i \beta)}+x(t-i \beta) \overline{y(t-i \beta)}) d t .
$$

Пространство Бергмана $\mathscr{A}_{2}^{\beta}(\mathbb{T})$ также является гильбертовым со скалярным произведением

$$
(x(\cdot), y(\cdot))_{\mathscr{A}_{2}^{\beta}(\mathbb{T})}=\frac{1}{4 \pi \beta} \int_{\mathbb{T}} d t \int_{-\beta}^{\beta} x(t+i \rho) \overline{y(t+i \rho)} d \rho .
$$

Система функций $\left\{e^{i j \cdot}\right\}_{j \in \mathbb{Z}}$ образует ортогональный базис в пространствах $\mathscr{H}_{2}^{\beta}(\mathbb{T})$ и $\mathscr{A}_{2}^{\beta}(\mathbb{T})$, при этом

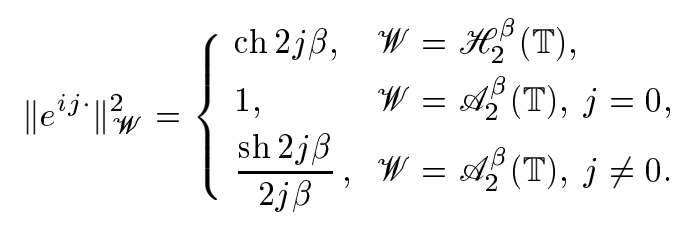


Тем самым, $x(\cdot) \in W=W_{2}^{r}(\mathbb{T}), H_{2}^{r, \beta}(\mathbb{T}), A_{2}^{r, \beta}(\mathbb{T})$ в том и только том случае, если

$$
x(t)=\sum_{j \in \mathbb{Z}} x_{j} e^{i j t}
$$

и

$$
\sum_{j \in \mathbb{Z}} \nu_{j}(W)\left|x_{j}\right|^{2} \leqslant 1
$$

где

$$
\nu_{j}(W)= \begin{cases}j^{2 r}, & W=W_{2}^{r}(\mathbb{T}), \\ j^{2 r} \operatorname{ch} 2 j \beta, & W=H_{2}^{r, \beta}(\mathbb{T}), \\ j^{2 r} \frac{\operatorname{sh} 2 j \beta}{2 j \beta}, & W=A_{2}^{r, \beta}(\mathbb{T}) .\end{cases}
$$

При этом в качестве $X$ рассматриваются пространства

$$
X=X^{\nu(W)}=\left\{x(\cdot) \in L_{2}(\mathbb{T}): \sum_{j \in \mathbb{Z}} \nu_{j}(W)\left|x_{j}\right|^{2}<\infty\right\} .
$$

Операторы мультипликаторного типа $T: X \rightarrow L_{2}(\mathbb{T})$, которые здесь изучаются, определяются следующим образом: если $y(\cdot)=T x(\cdot)$ и $\left\{x_{j}\right\}_{j \in \mathbb{Z}},\left\{y_{j}\right\}_{j \in \mathbb{Z}}-$ коэффициенты Фурье $x(\cdot)$ и $y(\cdot)$ соответственно, то $y_{j}=\gamma_{j} x_{j}, j \in \mathbb{Z}$, где $\left\{\gamma_{j}\right\}_{j \in \mathbb{Z}}$ - некоторая последовательность чисел. Ясно, что, например, оператору дифференцирования порядка $k>0$ соответствует последовательность $\gamma_{j}=(i j)^{k}, j \in \mathbb{Z}$.

Опишем, наконец, информационные отображения, которые будут рассматриваться.

1. Информация $I x(\cdot)=I_{\delta} x(\cdot)$ о функции $x(\cdot) \in W$ заключается в том, что мы располагаем числами $\left\{y_{j}\right\}_{j \in \mathbb{Z}}$ такими, что

$$
\sum_{j \in \mathbb{Z}}\left|x_{j}-y_{j}\right|^{2} \leqslant \delta^{2}
$$

где $\left\{x_{j}\right\}_{j \in \mathbb{Z}}-$ коэффициенты Фурье $x(\cdot)$ и $\delta>0$. Формально это означает, что если

$$
Y=l_{2}=\left\{z=\left\{z_{j}\right\}_{j \in \mathbb{Z}}:\|z\|_{l_{2}}^{2}=\sum_{j \in \mathbb{Z}}\left|z_{j}\right|^{2}<\infty\right\}
$$

$F: X \rightarrow Y$ - линейный оператор такой, что $F x(\cdot)=\left\{x_{j}\right\}_{j \in \mathbb{Z}}$, а $B Y-$ единичный шар в $Y$, то $I_{\delta} x(\cdot)=F x(\cdot)+\delta B Y$.

2. Информация $I x(\cdot)=I_{\delta}^{2 N+1} x(\cdot)$ о функции $x(\cdot) \in W$ заключается в том, что мы располагаем числами $\left\{y_{j}\right\}_{|j| \leqslant N}$ такими, что

$$
\sum_{|j| \leqslant N}\left|x_{j}-y_{j}\right|^{2} \leqslant \delta^{2}
$$

где $\left\{x_{j}\right\}_{|j| \leqslant N}-$ первые $2 N+1$ коэффициентов $\Phi$ урье $x(\cdot)$ и $\delta>0$. В данном случае $I_{\delta}^{2 N+1} x(\cdot)=F x(\cdot)+\delta B Y$, где

$$
Y=l_{2}^{2 N+1}=\left\{z=\left\{z_{j}\right\}_{|j| \leqslant N}:\|z\|_{l_{2}^{2 N+1}}^{2}=\sum_{|j| \leqslant N}\left|z_{j}\right|^{2}\right\},
$$

$F x(\cdot)=\left\{x_{j}\right\}_{|j| \leqslant N} \cdot$ 
3. Информация $I x(\cdot)=I_{\bar{\delta}}^{2 N+1} x(\cdot)$ о функции $x(\cdot) \in W$ заключается в том, что мы располагаем числами $\left\{y_{j}\right\}_{|j| \leqslant N}$ такими, что $\left|x_{j}-y_{j}\right| \leqslant \delta_{j},|j| \leqslant N$, где $\left\{x_{j}\right\}_{|j| \leqslant N}-$ первые $2 N+1$ коэффиициентов Фурье $x(\cdot), \bar{\delta}=\left\{\delta_{j}\right\}_{|j| \leqslant N}$ и $\delta_{j}>0$, $|j| \leqslant N$. Если

$$
Y=l_{\infty}^{2 N+1}=\left\{z=\left\{z_{j}\right\}_{|j| \leqslant N}:\|z\|_{l_{\infty}^{2 N+1}}=\sup _{|j| \leqslant N}\left|z_{j}\right|\right\}
$$

$F x(\cdot)=\left\{x_{j}\right\}_{|j| \leqslant N}$ И

$$
B(\bar{\delta})=\left\{z=\left\{z_{j}\right\}_{|j| \leqslant N}:\left|z_{j}\right| \leqslant \delta_{j},|j| \leqslant N\right\}
$$

то $I_{\bar{\delta}}^{2 N+1} x(\cdot)=F x(\cdot)+B(\bar{\delta})$.

При некоторых предположениях о последовательности $\nu=\left\{\nu_{j}\right\}_{j \in \mathbb{Z}}$, задающей класс $W$, и последовательности $\gamma=\left\{\gamma_{j}\right\}_{j \in \mathbb{Z}}$, задающей оператор $T$, для всех перечисленных информационных отображений мы находим величину погрешности оптимального восстановления и выписываем оптимальный метод восстановления оператора $T$ на классе $W$. В качестве следствия приводятся соответствующие утверждения для ряда конкретных классов гладких и аналитических функций.

\section{§2. Формулировка основных результатов}

Пусть даны последовательности $\nu=\left\{\nu_{j}\right\}_{j \in \mathbb{Z}}$ и $\gamma=\left\{\gamma_{j}\right\}_{j \in \mathbb{Z}}$. Положим $\mu_{j}=$ $\left|\gamma_{j}\right|^{2}, j \in \mathbb{Z}$. Будем предполагать, что выполнены следуюшие условия:

1) $\left\{\mu_{j}\right\}_{j \in \mathbb{Z}}$ и $\left\{\nu_{j}\right\}_{j \in \mathbb{Z}}$ четные последовательности (т.е. $\mu_{j}=\mu_{-j}$ и $\nu_{j}=\nu_{-j}$, $j \in \mathbb{Z})$ и $\mu_{0}=\nu_{0}=0$;

2) $\left\{\mu_{j}\right\}_{j \in \mathbb{N}},\left\{\nu_{j} \mu_{j}^{-1}\right\}_{j \in \mathbb{N}}$ - положительные возрастающие последовательности и $\nu_{j} \rightarrow \infty$ при $j \rightarrow \infty$;

3) при всех $\lambda_{1}, \lambda_{2}>0$ последовательность $\left\{-\mu_{j}+\lambda_{1}+\lambda_{2} \nu_{j}\right\}_{j \in \mathbb{Z}_{+}}$имеет не более двух перемен знака (при замене нулевых значений на любое из значений \pm 1$)$.

2.1. Восстановление по неточной информации о коэффициентах Фурье в метрике $l_{2}$. Задача (1) записывается в данном случае так:

$$
E\left(T, W, I_{\delta}\right)=\inf _{\varphi: l_{2} \rightarrow L_{2}(\mathbb{T})} \sup _{\substack{x(\cdot) \in W, y \in l_{2} \\\|F x(\cdot)-y\|_{l_{2}} \leqslant \delta}}\|T x(\cdot)-\varphi(y)(\cdot)\|_{L_{2}(\mathbb{T})}
$$

где $F x(\cdot)=\left\{x_{j}\right\}_{j \in \mathbb{Z}}-$ коэффициенты

ТЕОРема 1. Пусть последовательности $\left\{\mu_{j}\right\}_{j \in \mathbb{Z}} u\left\{\nu_{j}\right\}_{j \in \mathbb{Z}}$ удовлетворяют условиям 1)-3), ат и

$$
E\left(T, W, I_{\delta}\right)= \begin{cases}\sqrt{\frac{\mu_{1}}{\nu_{1}}}, & \delta \geqslant \nu_{1}^{-1 / 2}, \\ \sqrt{\delta^{2} \mu_{s}+\left(1-\delta^{2} \nu_{s}\right) \frac{\mu_{s+1}-\mu_{s}}{\nu_{s+1}-\nu_{s}}}, & \nu_{s+1}^{-1 / 2} \leqslant \delta<\nu_{s}^{-1 / 2}, s \geqslant 1 .\end{cases}
$$


При этом метод

$$
\widehat{\varphi}(y)(\cdot)=\sum_{j \in \mathbb{Z}} \gamma_{j}\left(1+\nu_{j} \frac{\mu_{s+1}-\mu_{s}}{\mu_{s} \nu_{s+1}-\mu_{s+1} \nu_{s}}\right)^{-1} y_{j} e^{i j}
$$

является оптимальным, если $\nu_{s+1}^{-1 / 2} \leqslant \delta<\nu_{s}^{-1 / 2}, s \geqslant 1$, а если $\delta \geqslant \nu_{1}^{-1 / 2}$, то $\widehat{\varphi}(y)(\cdot)=0$ - оптимальный метод.

Применим теорему 1 к задаче оптимального восстановления $k$-й производной (соответствуюший оператор будем обозначать через $D^{k}$ ) функции из класса $W=$ $W_{2}^{r}(\mathbb{T}), H_{2}^{r, \beta}(\mathbb{T}), A_{2}^{r, \beta}(\mathbb{T})$ по информации $I_{\delta}$. Нетрудно убедиться, что в этом случае для $\nu_{j}=\nu_{j}(W)$ и $\mu_{j}=j^{2 k}$ условия 1$)-3$ ) вьполнены (последнее из них вытекает из того, что при всех $\lambda_{1}, \lambda_{2}>0$ последовательность $\left\{\left(\lambda_{1}+\lambda_{2} \nu_{j}(W)\right) \mu_{j}^{-1}\right\}_{j \in \mathbb{N}}$ является выпуклой, т.е. ее вторая разность неотрицательна). Тем самым получаем

СЛЕДСТВИЕ 1. Для погрешности оптимального восстановления $k$-й производной функции из класса $W=W_{2}^{r}(\mathbb{T}), H_{2}^{r, \beta}(\mathbb{T}), A_{2}^{r, \beta}(\mathbb{T})$ по информации $I_{\delta}$ имеет место равенство

$$
E\left(D^{k}, W, I_{\delta}\right)=\nu_{1}^{-1 / 2}(W)
$$

nрu $\delta \geqslant \nu_{1}^{-1 / 2}(W) u$

$$
E\left(D^{k}, W, I_{\delta}\right)=\sqrt{\delta^{2} s^{2 k}+\left(1-\delta^{2} \nu_{s}(W)\right) \frac{(s+1)^{2 k}-s^{2 k}}{\nu_{s+1}(W)-\nu_{s}(W)}}
$$

nри $\nu_{s+1}^{-1 / 2}(W) \leqslant \delta<\nu_{s}^{-1 / 2}(W), s \geqslant 1$. При этом метод

$$
\widehat{\varphi}(y)(\cdot)=\sum_{|j| \geqslant 1}(i j)^{k}\left(1+\nu_{j}(W) \frac{(s+1)^{2 k}-s^{2 k}}{s^{2 k} \nu_{s+1}(W)-(s+1)^{2 k} \nu_{s}(W)}\right)^{-1} y_{j} e^{i j}
$$

является оптимальным, если $\nu_{s+1}^{-1 / 2}(W) \leqslant \delta<\nu_{s}^{-1 / 2}(W), s \geqslant 1$, аесли $\delta \geqslant \nu_{1}^{-1 / 2}(W)$, то $\widehat{\varphi}(y)(\cdot)=0$ - оптимальный метод.

Для восстановления самих функций $(k=0)$ из классов $W=W_{2}^{r}(\mathbb{T}), H_{2}^{r, \beta}(\mathbb{T})$, $A_{2}^{r, \beta}(\mathbb{T})$ имеет место следующий результат:

$$
E\left(\mathrm{Id}, W, I_{\delta}\right)=\delta,
$$

где Id - тождественный оператор, и

$$
\widehat{\varphi}(y)(\cdot)=\sum_{j \in \mathbb{Z}} y_{j} e^{i j}
$$

- оптимальный метод. 
2.2. Восстановление по неточной информации о коэффициентах Фурье в метрике $l_{2}^{2 N+1}$. В этом случае задача (1) имеет вид:

$$
E\left(T, W, I_{\delta}^{2 N+1}\right)=\inf _{\varphi: l_{2}^{2 N+1} \rightarrow L_{2}(\mathbb{T})} \sup _{\substack{x(\cdot) \in W, y \in l_{2}^{2 N+1} \\\|F x(\cdot)-y\|_{l_{2}^{2 N+1}}^{2 N} \leqslant \delta}}\|T x(\cdot)-\varphi(y)(\cdot)\|_{L_{2}(\mathbb{T})},
$$

где $F x(\cdot)=\left\{x_{j}\right\}_{|j| \leqslant N}-$ первые $2 N+1$ коэффициентов $\Phi$ урье $x(\cdot)$.

ТЕОРема 2. Пусть выполнены условия теоремы 1. Положсим

$$
s_{0}=s_{0}(N)=\min \left\{s \in \mathbb{N}: \frac{\mu_{s+1}-\mu_{s}}{\nu_{s+1}-\nu_{s}} \leqslant \frac{\mu_{N+1}}{\nu_{N+1}}\right\} .
$$

Тогда при $\delta \geqslant \nu_{s_{0}}^{-1 / 2}$

$$
E\left(T, W, I_{\delta}^{2 N+1}\right)=E\left(T, W, I_{\delta}\right)
$$

u метод

$$
\widehat{\varphi}(y)(\cdot)=\sum_{|j| \leqslant N} \gamma_{j}\left(1+\nu_{j} \frac{\mu_{s+1}-\mu_{s}}{\mu_{s} \nu_{s+1}-\mu_{s+1} \nu_{s}}\right)^{-1} y_{j} e^{i j}
$$

является оптимальным, если $\nu_{s+1}^{-1 / 2} \leqslant \delta<\nu_{s}^{-1 / 2}, 1 \leqslant s \leqslant s_{0}-1$, а если $\delta \geqslant \nu_{1}^{-1 / 2}$, то $\widehat{\varphi}(y)(\cdot)=0$ - оптимальный метод. При $0<\delta<\nu_{s_{0}}^{-1 / 2}$

$$
E\left(T, W, I_{\delta}^{2 N+1}\right)=\sqrt{\delta^{2} \mu_{s_{0}}+\left(1-\delta^{2} \nu_{s_{0}}\right) \frac{\mu_{N+1}}{\nu_{N+1}}}
$$

$u$

$$
\widehat{\varphi}(y)(\cdot)=\sum_{|j| \leqslant N} \gamma_{j}\left(1+\nu_{j} \frac{\mu_{N+1}}{\mu_{s_{0}} \nu_{N+1}-\mu_{N+1} \nu_{s_{0}}}\right)^{-1} y_{j} e^{i j}
$$

- оптимальный метод.

Нетрудно убедиться, что при $M \geqslant N$

$$
E\left(T, W, I_{\delta}^{2 N+1}\right) \geqslant E\left(T, W, I_{\delta}^{2 M+1}\right) \geqslant E\left(T, W, I_{\delta}\right)
$$

Поэтому из теоремы 2 вытекает, что при $\delta \geqslant \delta_{N}=\nu_{s_{0}}^{-1 / 2}$ увеличение числа коэффициентов Фурье, известных с той же погрешностью $\delta$, не приводит к уменьшению погрешности оптимального восстановления.

Тем самым при фиксированном уровне погрешности $\delta$ минимальное число первых коэффициентов Фурье (без учета нулевого, так как в силу условия $\mu_{0}=0$ он не используется в оптимальном методе), которые надо знать для максимально точного восстановления оператора $T$, равно $2 N_{0}$, где

$$
N_{0}=\min \left\{N \in \mathbb{N}: \delta_{N} \leqslant \delta\right\}
$$

Очевидным образом может быть сформулирован аналог следствия 1 для задачи восстановления $k$-й производной функции из класса $W=W_{2}^{r}(\mathbb{T}), H_{2}^{r, \beta}(\mathbb{T}), A_{2}^{r, \beta}(\mathbb{T})$ 
по информации $I_{\delta}^{2 N+1}$. Что касается восстановления самих функций из этих классов, то справедлив следуюший результат:

$$
E\left(\mathrm{Id}, W, I_{\delta}^{2 N+1}\right)=\sqrt{\delta^{2}+\nu_{N+1}^{-1}(W)}
$$

и

$$
\widehat{\varphi}(y)(\cdot)=\sum_{|j| \leqslant N}\left(1+\frac{\nu_{j}(W)}{\nu_{N+1}(W)}\right)^{-1} y_{j} e^{i j}
$$

- оптимальный метод.

2.3. Восстановление по неточной информации о коэффициентах Фурье в равномерной метрике. Задача (1) записывается в этом случае так:

$$
E\left(T, W, I_{\bar{\delta}}^{2 N+1}\right)=\inf _{\varphi: l_{\infty}^{2 N+1} \rightarrow L_{2}(\mathbb{T})} \sup _{\substack{x(\cdot) \in W, y \in l_{\infty}^{2 N+1} \\ F x(\cdot)-y \in B(\bar{\delta})}}\|T x(\cdot)-\varphi(y)(\cdot)\|_{L_{2}(\mathbb{T})}
$$

где $F x(\cdot)=\left\{x_{j}\right\}_{|j| \leqslant N}-$ первые $2 N+1$ коэффициентов Фурье $x(\cdot)$, а $B(\bar{\delta})-$ параллелепипед, определенный равенством (2).

Теорема 3. Пусть $\mu_{j}, \nu_{j}>0, j \in \mathbb{N},\left\{\nu_{j} \mu_{j}^{-1}\right\}_{j \in \mathbb{N}}-$ возрастающая последовательность, $\nu_{0}=0,\left\{\mu_{j}\right\}_{j \in \mathbb{Z}} u\left\{\nu_{j}\right\}_{j \in \mathbb{Z}}-$ четные последовательности, а $T$ и $W$ - соответствующие им оператор и класс. Положим

$$
p_{0}=p_{0}(\bar{\delta})=\max \left\{p \in \mathbb{Z}_{+}: \sum_{|j| \leqslant p} \nu_{j} \delta_{j}^{2}<1,0 \leqslant p \leqslant N\right\}
$$

Tогда

$$
E\left(T, W, I_{\bar{\delta}}^{2 N+1}\right)=\sqrt{\frac{\mu_{p_{0}+1}}{\nu_{p_{0}+1}}+\sum_{|j| \leqslant p_{0}}\left(\mu_{j}-\nu_{j} \frac{\mu_{p_{0}+1}}{\nu_{p_{0}+1}}\right) \delta_{j}^{2}}
$$

при этом метод

$$
\widehat{\varphi}(y)(\cdot)=\gamma_{0} y_{0}+\sum_{1 \leqslant|j| \leqslant p_{0}} \gamma_{j}\left(1-\frac{\mu_{p_{0}+1} \nu_{j}}{\nu_{p_{0}+1} \mu_{j}}\right) y_{j} e^{i j}
$$

является оптимальным.

Эта теорема также применима к задаче оптимального восстановления функций и их производных из классов $W=W_{2}^{r}(\mathbb{T}), H_{2}^{r, \beta}(\mathbb{T}), A_{2}^{r, \beta}(\mathbb{T})$ по информации $I_{\bar{\delta}}^{2 N+1}$. При этом, как и ранњше, для формулировки соответствующего результата надо положить в теореме $3 \mu_{j}=j^{2 k}, \nu_{j}=\nu_{j}(W), j \in \mathbb{Z}$. 


\section{§3. Доказательства}

Перед непосредственным доказательством сформулированных выше теорем отметим два утверждения общего характера и затем опишем схему, по которой эти теоремы будут доказываться.

Лемма 1. Пусть в задаче (1)

$$
\operatorname{gr} I=\{(x, y) \in X \times Y: x \in W, y \in I(x)\}
$$

является выпуклым центрально-симметричным множсеством. Тогда

$$
E(T, W, I) \geqslant \sup _{\substack{x \in W \\ x \in I^{-1}(0)}}\|T x\|_{Z}
$$

əде $I^{-1}(0)=\{x \in W: 0 \in I(x)\}$.

ДокАЗАТЕЛЬСТво. Для любого метода $\varphi$ при всех $x \in W$ таких, что $x \in I^{-1}(0)$, имеем

$$
2\|T x\|_{Z} \leqslant\|T x-\varphi(0)\|_{Z}+\|T(-x)-\varphi(0)\|_{Z} \leqslant 2 e(T, W, I, \varphi) .
$$

Следовательно, для любого метода $\varphi$

$$
e(T, W, I, \varphi) \geqslant \sup _{\substack{x \in W \\ x \in I^{-1}(0)}}\|T x\|_{Z}
$$

откуда сразу же вытекает доказываемая оценка.

Лемма 2. Пусть $X, Y, Z, W, T$ и $I$ me жсе, что и в задаче (1), $Y_{0}$ линейное пространство с полускалярным произведением $(\cdot, \cdot)_{Y_{0}}$ и соответствующей полунормой $\|\cdot\|_{Y_{0}}, I_{0}: X \rightarrow Y_{0}, S: Y \rightarrow Y_{0}$ - линейнье операторь $u$

$$
\operatorname{gr} I \subset\left\{(x, y) \in X \times Y:\left\|I_{0} x-S y\right\|_{Y_{0}} \leqslant 1\right\}
$$

Пусть, далее, $\psi: Y \rightarrow X-$ отображение, сопоставляющее $у \in Y$ решение экстремальной задачи

$$
\left\|I_{0} x-S y\right\|_{Y_{0}}^{2} \rightarrow \min , \quad x \in X
$$

Тогда для погрешности метода $\widehat{\varphi}=T \circ \psi$ справедлива оченка

$$
e(T, W, I, \widehat{\varphi}) \leqslant \sup _{\left\|I_{0} x\right\|_{Y_{0}} \leqslant 1}\|T x\|_{Z}
$$


ДокАЗАТЕльство. Нетрудно проверить, что для того чтобы $\widehat{x} \in X$ было решением задачи (7), необходимо и достаточно выполнение соотношения

$$
\left(I_{0} \widehat{x}-S y, I_{0} x\right)_{Y_{0}}=0 \quad \forall x \in X .
$$

Пусть $(x, y) \in \operatorname{gr} I$ (т.е. $x \in W$ и $y \in I(x))$, тогда по условию $\left\|I_{0} x-S y\right\|_{Y_{0}} \leqslant 1$. Так как $\psi(y)$ - решение (7), то выполнено (8) (с заменой $\widehat{x}$ на $\psi(y))$ и поэтому

$$
\left\|I_{0} x-S y\right\|_{Y_{0}}^{2}=\left\|I_{0} x-I_{0}(\psi(y))\right\|_{Y_{0}}^{2}+\left\|I_{0}(\psi(y))-S y\right\|_{Y_{0}}^{2} .
$$

Следовательно,

$$
\left\|I_{0}(x-\psi(y))\right\|_{Y_{0}} \leqslant\left\|I_{0} x-S y\right\|_{Y_{0}} \leqslant 1
$$

и, значит,

$$
\|T x-\widehat{\varphi}(y)\|_{Z}=\|T x-T(\psi(y))\|_{Z}=\|T(x-\psi(y))\|_{Z} \leqslant \sup _{\left\|I_{0} h\right\|_{Y_{0}} \leqslant 1}\|T h\|_{Z} .
$$

Дальнейший план доказательства теорем таков. Оценка снизу (лемма 1) и оценка сверху (лемма 2) для погрешности оптимального восстановления представляют собой значения экстремальных задач. В силу определений класса $W$ и оператора $T$ эти задачи редуцируются к задачам выпуклого программирования. Используя стандартные методы выпуклой оптимизации, мы находим значение задачи, соответствующей оценке снизу, и показываем, что оно совпадает со значением задачи, соответствующей оценке сверху при некоторых $Y_{0}, I_{0}$ и $S$. Это означает в силу лемм 1 и 2 , что данное значение есть погрешность оптимального восстановления, а метод из леммы 2 - оптимальный метод восстановления (при этом он оказывается линейньм).

ДокаЗАтЕЛЬСтво теоремы 1. 1. Оценка снизу. Согласно лемме 1 и (3) мы должны найти значение следующей задачи:

$$
\|T x(\cdot)\|_{L_{2}(\mathbb{T})} \rightarrow \max , \quad\|F x(\cdot)\|_{l_{2}} \leqslant \delta, \quad x(\cdot) \in W .
$$

Переходя к образам Фурье, получаем в силу равенства Парсеваля и определений оператора $T$ и класса $W$, что эта задача (с заменой $\|T x(\cdot)\| L_{L_{2}(\mathbb{T})}$ на $\|T x(\cdot)\|_{L_{2}(\mathbb{T})}^{2}$ ) может быть записана следующим образом

$$
\sum_{j \in \mathbb{Z}} \mu_{j}\left|x_{j}\right|^{2} \rightarrow \max , \quad \sum_{j \in \mathbb{Z}}\left|x_{j}\right|^{2} \leqslant \delta^{2}, \quad \sum_{j \in \mathbb{Z}} \nu_{j}\left|x_{j}\right|^{2} \leqslant 1 .
$$

Обозначая $u_{j}=\left|x_{j}\right|^{2}, j \in \mathbb{Z}$, задачу (10) перепишем в виде

$$
\sum_{j \in \mathbb{Z}} \mu_{j} u_{j} \rightarrow \max , \quad \sum_{j \in \mathbb{Z}} u_{j} \leqslant \delta^{2}, \quad \sum_{j \in \mathbb{Z}} \nu_{j} u_{j} \leqslant 1, \quad u_{j} \geqslant 0 .
$$

Это задача выпуклого (и даже линейного) программирования. Сопоставим ей функцию Лагранжа $\left(u=\left\{u_{j}\right\}_{j \in \mathbb{Z}}\right)$

$$
\mathscr{L}=\mathscr{L}\left(u, \lambda_{0}, \lambda_{1}, \lambda_{2}\right)=\sum_{j \in \mathbb{Z}}\left(\lambda_{0} \mu_{j}+\lambda_{1}+\lambda_{2} \nu_{j}\right) u_{j},
$$


где $\lambda_{0} \leqslant 0, \lambda_{1}, \lambda_{2} \geqslant 0$ - множители Лагранжа. Согласно теореме Куна-Таккера если $\widehat{u}=\left\{\widehat{u}_{j}\right\}_{j \in \mathbb{Z}}-$ решение задачи (11), то найдутся такие множители Лагранжа $\widehat{\lambda}_{0} \leqslant 0, \widehat{\lambda}_{1}, \widehat{\lambda}_{2} \geqslant 0$, не все равные нулю, что выполнены условия

$$
\begin{aligned}
& \text { (a) } \min _{u_{j} \geqslant 0} \mathscr{L}\left(u, \widehat{\lambda}_{0}, \widehat{\lambda}_{1}, \widehat{\lambda}_{2}\right)=\mathscr{L}\left(\widehat{u}, \widehat{\lambda}_{0}, \widehat{\lambda}_{1}, \widehat{\lambda}_{2}\right), \\
& \text { (b) } \widehat{\lambda}_{1}\left(\sum_{j \in \mathbb{Z}} \widehat{u}_{j}-\delta^{2}\right)=0, \widehat{\lambda}_{2}\left(\sum_{j \in \mathbb{Z}} \nu_{j} \widehat{u}_{j}-1\right)=0 .
\end{aligned}
$$

Если для допустимой в (11) последовательности $\widehat{u}=\left\{\widehat{u}_{j}\right\}_{j \in \mathbb{Z}}$ выполнены условия (a) и (b) с $\widehat{\lambda}_{0}<0$, то $\widehat{u}$ - решение задачи (11).

Последнее утверждение может быть легко проверено. Действительно, пусть $u=\left\{u_{j}\right\}_{j \in \mathbb{Z}}-$ допустимая последовательность в (11). Тогда, учитывая (a) и (b), будем иметь

$$
\begin{aligned}
\widehat{\lambda}_{0} \sum_{j \in \mathbb{Z}} \mu_{j} u_{j} & \geqslant \widehat{\lambda}_{0} \sum_{j \in \mathbb{Z}} \mu_{j} u_{j}+\widehat{\lambda}_{1}\left(\sum_{j \in \mathbb{Z}} u_{j}-\delta^{2}\right)+\widehat{\lambda}_{2}\left(\sum_{j \in \mathbb{Z}} \nu_{j} u_{j}-1\right) \\
& \stackrel{(\mathrm{a})}{\geqslant} \widehat{\lambda}_{0} \sum_{j \in \mathbb{Z}} \mu_{j} \widehat{u}_{j}+\widehat{\lambda}_{1}\left(\sum_{j \in \mathbb{Z}} \widehat{u}_{j}-\delta^{2}\right)+\widehat{\lambda}_{2}\left(\sum_{j \in \mathbb{Z}} \nu_{j} \widehat{u}_{j}-1\right) \\
& \stackrel{(\mathrm{b})}{=} \widehat{\lambda}_{0} \sum_{j \in \mathbb{Z}} \mu_{j} \widehat{u}_{j}
\end{aligned}
$$

т.е. $\widehat{u}=\left\{\widehat{u}_{j}\right\}_{j \in \mathbb{Z}}-$ решение задачи (11).

Мы предъявим сейчас такие $\widehat{\lambda}_{1}, \widehat{\lambda}_{2} \geqslant 0$ и допустимую последовательность $\widehat{u}=$

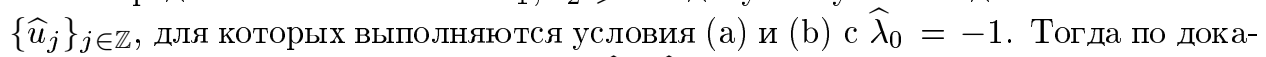
занному $\widehat{u}$ - решение задачи (11). Вид $\widehat{\lambda}_{1}, \widehat{\lambda}_{2}$ и $\widehat{u}$ следует из анализа соотношений (a) и (b). Действительно, последовательность $f_{j}=-\mu_{j}+\widehat{\lambda}_{1}+\widehat{\lambda}_{2} \nu_{j}, j \in \mathbb{Z}$, должна быть неотрицательной и решение должно быть сосредоточено в нулях $\left\{f_{j}\right\}_{j \in \mathbb{Z}}$. Вследствие условия на число перемен знака этой последовательности (условие 3$)$ ) положительные нули $\left\{f_{j}\right\}_{j \in \mathbb{Z}}$ могут идти только подряд. Эти наблюдения позволяют предъявить соответствующие $\widehat{\lambda}_{1}, \widehat{\lambda}_{2}$ и $\widehat{u}$.

Пусть сначала $0<\delta<\nu_{1}^{-1 / 2}$. Так как $\nu_{j} \rightarrow \infty$ при $j \rightarrow \infty$, найдется $s \geqslant 1$, для которого $\nu_{s+1}^{-1 / 2} \leqslant \delta<\nu_{s}^{-1 / 2}$. Найдем $\widehat{\lambda}_{1}$ и $\widehat{\lambda}_{2}$ из условия, что $f_{s}=f_{s+1}=0$. Тогда получим

$$
\widehat{\lambda}_{1}=\frac{\mu_{s} \nu_{s+1}-\mu_{s+1} \nu_{s}}{\nu_{s+1}-\nu_{s}}, \quad \widehat{\lambda}_{2}=\frac{\mu_{s+1}-\mu_{s}}{\nu_{s+1}-\nu_{s}} .
$$

Из предположений о последовательностях $\left\{\mu_{j}\right\}_{j \in \mathbb{Z}}$ и $\left\{\nu_{j}\right\}_{j \in \mathbb{Z}}$ следует, что $\widehat{\lambda}_{1}, \widehat{\lambda}_{2}$ $>0$. С полученньпи $\widehat{\lambda}_{1}$ и $\widehat{\lambda}_{2}$ последовательность $\left\{f_{j}\right\}_{j \in \mathbb{Z}}$ неотрицательна в силу условия на число перемен знака этой последовательности. Теперь найдем последовательность $\widehat{u}$ из условия, что она сосредоточена в точках $s$ и $s+1$ и вьполняются условия (b), т.е. $u_{s}+u_{s+1}=\delta^{2}$ и $\nu_{s} u_{s}+\nu_{s+1} u_{s+1}=1$. Отсюда получаем, что

$$
\widehat{u}_{s}=\frac{\delta^{2} \nu_{s+1}-1}{\nu_{s+1}-\nu_{s}}, \quad \widehat{u}_{s+1}=\frac{1-\delta^{2} \nu_{s}}{\nu_{s+1}-\nu_{s}}
$$


$\left(\widehat{u}_{s} \geqslant 0, \widehat{u}_{s+1}>0\right.$ в силу условия на $\left.\delta\right)$. Положим $\widehat{u}_{j}=0$ при $j \neq s, s+1$. Тем самым $\widehat{u}$ - допустимая последовательность. Условия (a) и (b) с найденными $\widehat{\lambda}_{1}, \widehat{\lambda}_{2}, \widehat{u}$ выполняются при $\widehat{\lambda}_{0}=-1$, и поэтому $\widehat{u}$ - решение задачи (11).

Пусть теперь $\delta \geqslant \nu_{1}^{-1 / 2}$. Положим $\widehat{\lambda}_{1}=0, \widehat{\lambda}_{2}=\mu_{1} \nu_{1}^{-1}$. Тогда $f_{j}=0$ при $|j| \leqslant 1$ и $f_{j} \geqslant 0$ при $|j| \geqslant 2$. Положим $\widehat{u}_{1}=\nu_{1}^{-1}$ и $\widehat{u}_{j}=0$ при $j \neq 1$. Так как $\widehat{u}_{1}=\nu_{1} \leqslant \delta^{2}$, то $\widehat{u}$ - допустимая последовательность. Условия (а) и (b) очевидньм образом выполняются с $\widehat{\lambda}_{0}=-1$, и, следовательно, $\widehat{u}-$ решение задачи $(11)$.

Итак, найдено решение задачи (11) для всех $\delta>0$, а значит, решение задач $(10)$ и (9). Подставляя $\widehat{u}$ в максимизируемый функционал в (11) и извлекая квадратный корень, получаем значение задачи (9), которое дает оценку снизу для погрешности оптимального восстановления

$$
E\left(T, W, I_{\delta}\right) \geqslant \begin{cases}\sqrt{\frac{\mu_{1}}{\nu_{1}}}, & \delta \geqslant \nu_{1}^{-1 / 2}, \\ \sqrt{\delta^{2} \mu_{s}+\left(1-\delta^{2} \nu_{s}\right) \frac{\mu_{s+1}-\mu_{s}}{\nu_{s+1}-\nu_{s}}}, & \nu_{s+1}^{-1 / 2} \leqslant \delta<\nu_{s}^{-1 / 2}, s \geqslant 1 .\end{cases}
$$

2. Оценка сверху. Для найденных вьше $\widehat{\lambda}_{1}$ и $\widehat{\lambda}_{2}$ положим $\hat{\lambda}=\widehat{\lambda}_{1} \delta^{2}+\widehat{\lambda}_{2}$ и $\widehat{\alpha}=\widehat{\lambda}_{2}\left(\widehat{\lambda}_{1} \delta^{2}+\widehat{\lambda}_{2}\right)^{-1}$. Условие (а) для найденного решения $\widehat{u}$ задачи (11) может быть переписано в виде (с $\left.\widehat{\lambda}_{0}=-1\right)$

$\left(\mathrm{a}_{1}\right) \min _{u_{j} \geqslant 0} \sum_{j \in \mathbb{Z}}\left(-\mu_{j}+\widehat{\lambda}\left((1-\widehat{\alpha}) \delta^{-2}+\widehat{\alpha} \nu_{j}\right)\right) u_{j}=\sum_{j \in \mathbb{Z}}\left(-\mu_{j}+\widehat{\lambda}\left((1-\widehat{\alpha}) \delta^{-2}+\widehat{\alpha} \nu_{j}\right)\right) \widehat{u}_{j}$.

Кроме того, легко проверить, что выполняется соотношение

$$
\left(\mathrm{b}_{1}\right)(1-\widehat{\alpha}) \delta^{-2} \sum_{j \in \mathbb{Z}} \widehat{u}_{j}+\widehat{\alpha} \sum_{j \in \mathbb{Z}} \nu_{j} \widehat{u}_{j}=1
$$

По тем же соображениям, что и ранњше, эти условия достаточны для того, чтобы $\widehat{u}$ было решением задачи

$$
\sum_{j \in \mathbb{Z}} \mu_{j} u_{j} \rightarrow \max , \quad(1-\widehat{\alpha}) \delta^{-2} \sum_{j \in \mathbb{Z}} u_{j}+\widehat{\alpha} \sum_{j \in \mathbb{Z}} \nu_{j} u_{j} \leqslant 1, \quad u_{j} \geqslant 0 .
$$

Таким образом, значения задач (11) и (14) совпадают.

Покажем, что к задаче (14) может быть редуцирована задача из леммы 2. Действительно, пусть $Y_{0}=l_{2} \times l^{\nu}$, где

$$
l^{\nu}=\left\{z=\left\{z_{j}\right\}_{j \in \mathbb{Z}}: \sum_{j \in \mathbb{Z}} \nu_{j}\left|z_{j}\right|^{2}<\infty\right\}
$$

Определим на $Y_{0}$ полускалярное произведение

$$
\left((x, z),\left(x^{\prime}, z^{\prime}\right)\right)_{Y_{0}}=(1-\widehat{\alpha}) \delta^{-2} \sum_{j \in \mathbb{Z}} x_{j} \bar{x}_{j}^{\prime}+\widehat{\alpha} \sum_{j \in \mathbb{Z}} \nu_{j} z_{j} \bar{z}_{j}^{\prime}
$$


Пусть, далее, оператор $I_{0}: X \rightarrow Y_{0}$ определен равенством $I_{0} x(\cdot)=(F x(\cdot), F x(\cdot))$, a $S: l_{2} \rightarrow Y_{0}$ - равенством $S y=(y, 0)$. Если $x(\cdot) \in W$ и $\|F x(\cdot)-y\|_{l_{2}} \leqslant \delta$, т.е.

$$
\sum_{j \in \mathbb{Z}} \nu_{j}\left|x_{j}\right|^{2} \leqslant 1, \quad \sum_{j \in \mathbb{Z}}\left|x_{j}-y_{j}\right|^{2} \leqslant \delta^{2}
$$

то, очевидно,

$$
\left\|I_{0} x(\cdot)-S y\right\|_{Y_{0}}^{2}=(1-\widehat{\alpha}) \delta^{-2} \sum_{j \in \mathbb{Z}}\left|x_{j}-y_{j}\right|^{2}+\widehat{\alpha} \sum_{j \in \mathbb{Z}} \nu_{j}\left|x_{j}\right|^{2} \leqslant 1
$$

Согласно лемме 2 квадрат значения погрешности оптимального восстановления не превосходит значения задачи

$$
\|T x(\cdot)\|_{L_{2}(\mathbb{T})}^{2} \rightarrow \max , \quad\left\|I_{0} x(\cdot)\right\|_{Y_{0}}^{2} \leqslant 1
$$

которая после перехода к коэффициентам Фурье (по равенству Парсеваля) в максимизируемом функционале и замены $\left|x_{j}\right|^{2}$ на $u_{j}$ становится в точности задачей (14).

3. Оптимальный метод. Из леммы 2 вытекает, что оптимальный метод имеет вид $\widehat{\varphi}=T \circ \psi$, где коэффициенты Фурье $\left\{\psi_{j}\right\}_{j \in \mathbb{Z}}$ функции $\psi=\psi(y)$ есть решение экстремальной задачи

$$
(1-\widehat{\alpha}) \delta^{-2} \sum_{j \in \mathbb{Z}}\left|x_{j}-y_{j}\right|^{2}+\widehat{\alpha} \sum_{j \in \mathbb{Z}} \nu_{j}\left|x_{j}\right|^{2} \rightarrow \min , \quad x(\cdot) \in X
$$

Нетрудно убедиться, что

$$
\psi_{j}=\frac{(1-\widehat{\alpha}) \delta^{-2}}{(1-\widehat{\alpha}) \delta^{-2}+\widehat{\alpha} \nu_{j}} y_{j}, \quad j \in \mathbb{Z}
$$

Подставляя сюда выражение для $\widehat{\alpha}$, получаем требуемый результат.

Оценка снизу в (4) сразу следует из леммы 1 , а оптимальность метода (5) проверяется непосредственно.

ДоказАТЕЛЬСтво ТЕОРЕмЫ 2. Прежде всего отметим, что определение числа $s_{0}$ равенством (6) корректно, так как из возрастания последовательности $\left\{\nu_{j} \mu_{j}^{-1}\right\}_{j \in \mathbb{N} \text { вытекает неравенство }}$

$$
\frac{\mu_{N+1}-\mu_{N}}{\nu_{N+1}-\nu_{N}} \leqslant \frac{\mu_{N+1}}{\nu_{N+1}}
$$

Тем самым $1 \leqslant s_{0} \leqslant N$.

Остановимся только на решении экстремальной задачи

$$
\|T x(\cdot)\|_{L_{2}(\mathbb{T})} \rightarrow \max , \quad\|F x(\cdot)\|_{l_{2}^{2 N+1}} \leqslant \delta, \quad x(\cdot) \in W
$$


поскольку все остальные рассуждения в значительной мере повторяют доказательство теоремы 1. Переходя к коэффициентам Фурье и обозначая квадрат их модулей через $u_{j}$, приходим к эквивалентной задаче

$$
\sum_{j \in \mathbb{Z}} \mu_{j} u_{j} \rightarrow \max , \quad \sum_{|j| \leqslant N} u_{j} \leqslant \delta^{2}, \quad \sum_{j \in \mathbb{Z}} \nu_{j} u_{j} \leqslant 1, \quad u_{j} \geqslant 0
$$

Для решения этой задачи (так же как и для решения задачи (11)) достаточно предъявить такие $\widehat{\lambda}_{1}, \widehat{\lambda}_{2} \geqslant 0$ и допустимую последовательность $\left\{\widehat{u}_{j}\right\}_{j \in \mathbb{Z}}$, для которых при всех $u_{j} \geqslant 0, j \in \mathbb{Z}$,

$$
\text { (a) } \sum_{j \in \mathbb{Z}}\left(-\mu_{j}+\widehat{\lambda}_{1} \chi_{j}+\widehat{\lambda}_{2} \nu_{j}\right) u_{j} \geqslant \sum_{j \in \mathbb{Z}}\left(-\mu_{j}+\widehat{\lambda}_{1} \chi_{j}+\widehat{\lambda}_{2} \nu_{j}\right) \widehat{u}_{j}
$$

где

$$
\chi_{j}= \begin{cases}1, & |j| \leqslant N \\ 0, & |j|>N\end{cases}
$$

и, кроме того,

$$
\left(\mathrm{b}_{2}\right) \widehat{\lambda}_{1}\left(\sum_{|j| \leqslant N} \widehat{u}_{j}-\delta^{2}\right)=0, \quad \widehat{\lambda}_{2}\left(\sum_{j \in \mathbb{Z}} \nu_{j} \widehat{u}_{j}-1\right)=0
$$

Пусть $\nu_{s+1}^{-1 / 2} \leqslant \delta<\nu_{s}^{-1 / 2}$ и $1 \leqslant s \leqslant s_{0}-1$. Определим $\widehat{\lambda}_{1}$ и $\widehat{\lambda}_{2}$ из условий

$$
\begin{gathered}
-\mu_{s}+\widehat{\lambda}_{1}+\widehat{\lambda}_{2} \nu_{s}=0 \\
-\mu_{s+1}+\widehat{\lambda}_{1}+\widehat{\lambda}_{2} \nu_{s+1}=0
\end{gathered}
$$

Тогда для $\widehat{\lambda}_{1}$ и $\widehat{\lambda}_{2}$ справедливы равенства (12), из которых следует, что $\widehat{\lambda}_{1}, \widehat{\lambda}_{2}>0$. В силу того что последовательность $\left\{-\mu_{j}+\widehat{\lambda}_{1}+\widehat{\lambda}_{2} \nu_{j}\right\}_{j \in \mathbb{Z}_{+}}$имеет не более двух перемен знака, получаем

$$
-\mu_{j}+\widehat{\lambda}_{1}+\widehat{\lambda}_{2} \nu_{j} \geqslant 0, \quad|j| \leqslant N
$$

Из возрастания последовательности $\left\{\nu_{j} \mu_{j}^{-1}\right\}_{j \in \mathbb{N}}$ и того, что $s<s_{0}$, при $j \geqslant N+1$ имеем

$$
\frac{\mu_{j}}{\nu_{j}} \leqslant \frac{\mu_{N+1}}{\nu_{N+1}}<\frac{\mu_{s+1}-\mu_{s}}{\nu_{s+1}-\nu_{s}}=\widehat{\lambda}_{2}
$$

Отсюда $-\mu_{j}+\widehat{\lambda}_{2} \nu_{j}>0$ при $|j| \geqslant N+1$. Определив $\widehat{u}_{s}$ и $\widehat{u}_{s+1}$ равенствами (13) и положив $\widehat{u}_{j}=0, j \neq s, s+1$, получим, что $\left\{\widehat{u}_{j}\right\}_{j \in \mathbb{Z}}-$ допустимая последовательность, для которой выполняются условия $\left(\mathrm{a}_{2}\right)$ и $\left(\mathrm{b}_{2}\right)$. Следовательно, $\left\{\widehat{u}_{j}\right\}_{j \in \mathbb{Z}}-$ решение задачи (16).

Пусть теперь $0<\delta<\nu_{s_{0}}^{-1 / 2}$. Положим

$$
\widehat{\lambda}_{1}=\mu_{s_{0}}-\frac{\mu_{N+1}}{\nu_{N+1}} \nu_{s_{0}}, \quad \widehat{\lambda}_{2}=\frac{\mu_{N+1}}{\nu_{N+1}} .
$$


Из определения $s_{0}$ имеем

$$
\frac{\mu_{s_{0}}-\mu_{s_{0}-1}}{\nu_{s_{0}}-\nu_{s_{0}-1}}>\frac{\mu_{N+1}}{\nu_{N+1}} .
$$

Таким образом, $-\mu_{j}+\widehat{\lambda}_{1}+\widehat{\lambda}_{2} \nu_{j} \geqslant 0$ для $j=0, s_{0}-1$ и $-\mu_{s_{0}}+\widehat{\lambda}_{1}+\widehat{\lambda}_{2} \nu_{s_{0}}=0$. Из условия на число перемен знака получаем, что $-\mu_{j}+\widehat{\lambda}_{1}+\widehat{\lambda}_{2} \nu_{j} \geqslant 0$ при всех $|j| \leqslant s_{0}$. Если $s_{0}<N$, то в силу определения $s_{0}$

$$
\frac{\mu_{s_{0}+1}-\mu_{s_{0}}}{\nu_{s_{0}+1}-\nu_{s_{0}}} \leqslant \frac{\mu_{N+1}}{\nu_{N+1}}
$$

т.е. $-\mu_{s_{0}+1}+\widehat{\lambda}_{1}+\widehat{\lambda}_{2} \nu_{s_{0}+1} \geqslant 0$. Тогда из условия на число перемен знака $-\mu_{j}+$ $\widehat{\lambda}_{1}+\widehat{\lambda}_{2} \nu_{j} \geqslant 0$ при всех $|j| \leqslant N$. Если $|j|>N$, то

$$
-\mu_{j}+\widehat{\lambda}_{2} \nu_{j}=\mu_{j} \frac{\mu_{N+1}}{\nu_{N+1}}\left(\frac{\nu_{j}}{\mu_{j}}-\frac{\nu_{N+1}}{\mu_{N+1}}\right) \geqslant 0 .
$$

Тем самым доказано, что при всех $j \in \mathbb{Z}$

$$
-\mu_{j}+\widehat{\lambda}_{1} \chi_{j}+\widehat{\lambda}_{2} \nu_{j} \geqslant 0
$$

Положив

$$
\widehat{u}_{s_{0}}=\delta^{2}, \quad \widehat{u}_{N+1}=\frac{1-\delta^{2} \nu_{s_{0}}}{\nu_{N+1}}, \quad \widehat{u}_{j}=0, \quad j \neq s_{0}, N+1,
$$

легко убедиться, что $\left\{\widehat{u}_{j}\right\}_{j \in \mathbb{Z}}-$ допустимая последовательность, для которой выполнены условия $\left(\mathrm{a}_{2}\right)$ и $\left(\mathrm{b}_{2}\right)$.

Случай $\delta \geqslant \nu_{1}^{-1 / 2}$ рассматривается точно так же, как и в доказательстве теоремы 1.

Для восстановления самой функции из класса $W=W_{2}^{r}(\mathbb{T}), H_{2}^{r, \beta}(\mathbb{T}), A_{2}^{r, \beta}(\mathbb{T})$ теорему 2 формально применить нельзя $\left(\mu_{j}=1, j \in \mathbb{Z}\right.$, и тем самым не вьполнены условия теоремы относительно этой последовательности). Тем не менее, сама схема доказательства остается прежней.

ДоКАЗАТЕЛЬСТво ТЕОРЕмЫ 3. 1. Оценка снизу. Из леммы 1 получаем, что погрешность оптимального восстановления оценивается снизу значением задачи

$$
\|T x(\cdot)\|_{L_{2}(\mathbb{T})} \rightarrow \max , \quad\left|x_{j}\right| \leqslant \delta_{j}, \quad|j| \leqslant N, \quad x(\cdot) \in W,
$$

где $\left\{x_{j}\right\}_{|j| \leqslant N}-$ первые $2 N+1$ коэффициентов Фурье функции $x(\cdot)$. Полагая $u_{j}=$ $\left|x_{j}\right|^{2}, j \in \mathbb{Z}$, приходим к эквивалентной задаче

$$
\sum_{j \in \mathbb{Z}} \mu_{j} u_{j} \rightarrow \max , \quad \sum_{j \in \mathbb{Z}} \nu_{j} u_{j} \leqslant 1, \quad 0 \leqslant u_{j} \leqslant \delta_{j}^{2}, \quad|j| \leqslant N .
$$

Для нахождения решения этой задачи достаточно найти такие $\widehat{\lambda} \geqslant 0, \hat{\lambda}_{j} \geqslant 0$, $|j| \leqslant N$, и допустимую последовательность $\left\{\widehat{u}_{j}\right\}_{j \in \mathbb{Z}}$, для которых при всех $u_{j} \geqslant 0$, $j \in \mathbb{Z}$

$$
\text { (a) } \sum_{j \in \mathbb{Z}}\left(-\mu_{j}+\widehat{\lambda} \nu_{j}+\widehat{\lambda}_{j} \chi_{j}\right) u_{j} \geqslant \sum_{j \in \mathbb{Z}}\left(-\mu_{j}+\widehat{\lambda} \nu_{j}+\widehat{\lambda}_{j} \chi_{j}\right) \widehat{u}_{j}
$$


И

$$
\left(\mathrm{b}_{3}\right) \hat{\lambda}\left(\sum_{j \in \mathbb{Z}} \nu_{j} \widehat{u}_{j}-1\right)=0, \widehat{\lambda}_{j}\left(\widehat{u}_{j}-\delta_{j}^{2}\right)=0,|j| \leqslant N .
$$

Положим $\widehat{\lambda}=\frac{\mu_{p_{0}+1}}{\nu_{p_{0}+1}}$,

$$
\widehat{\lambda}_{j}= \begin{cases}\mu_{j}-\frac{\mu_{p_{0}+1}}{\nu_{p_{0}+1}} \nu_{j}, & |j| \leqslant p_{0} \\ 0, & p_{0}+1 \leqslant|j| \leqslant N\end{cases}
$$

Последовательность $\left\{\widehat{u}_{j}\right\}_{j \in \mathbb{Z}}$ определим равенством

$$
\widehat{u}_{j}= \begin{cases}\delta_{j}^{2}, & |j| \leqslant p_{0}, \\ \delta_{j}^{2} \frac{1-\sum_{|k| \leqslant p_{0}} \nu_{k} \delta_{k}^{2}}{\left(\delta_{p_{0}+1}^{2}+\delta_{-p_{0}-1}^{2}\right) \nu_{p_{0}+1}}, & |j|=p_{0}+1, \\ 0, & |j|>p_{0}+1 .\end{cases}
$$

Из определения $p_{0}$ следует, что последовательность $\widehat{u}=\left\{\widehat{u}_{j}\right\}_{j \in \mathbb{Z}}$ допустимая. Кроме того, $-\mu_{j}+\widehat{\lambda} \nu_{j}+\widehat{\lambda}_{j}=0$ при $|j| \leqslant p_{0}$. В силу возрастания последовательности $\left\{\nu_{j} \mu_{j}^{-1}\right\}_{j \in \mathbb{N}}$ при $|j| \geqslant p_{0}+1$ имеем $-\mu_{j}+\widehat{\lambda} \nu_{j} \geqslant 0$. Тем самым выполнено условие $\left(\mathrm{a}_{3}\right)$. Легко убедиться, что условие $\left(\mathrm{b}_{3}\right)$ также выполнено. Таким образом, $\widehat{u}$ - решение задачи (17). Подставляя $\widehat{u}$ в максимизируемый функционал и извлекая квадратный корень, получаем

$$
E\left(T, W^{\nu}, I_{\bar{\delta}}^{2 N+1}\right) \geqslant \sqrt{\frac{\mu_{p_{0}+1}}{\nu_{p_{0}+1}}+\sum_{|j| \leqslant p_{0}}\left(\mu_{j}-\nu_{j} \frac{\mu_{p_{0}+1}}{\nu_{p_{0}+1}}\right) \delta_{j}^{2}}
$$

2. Оценка сверху. Для найденных выше $\widehat{\lambda}_{\text {и }} \widehat{\lambda}_{j},|j| \leqslant N$, положим

$$
\lambda=\widehat{\lambda}+\sum_{|j| \leqslant N} \widehat{\lambda}_{j} \delta_{j}^{2}, \quad \alpha=\frac{\widehat{\lambda}}{\lambda}, \quad \alpha_{j}=\frac{\widehat{\lambda}_{j} \delta_{j}^{2}}{\lambda}, \quad|j| \leqslant N
$$

Тогда условие (аз) может быть переписано в виде

$$
\left(\mathrm{a}_{4}\right) \sum_{j \in \mathbb{Z}}\left(-\mu_{j}+\lambda\left(\alpha \nu_{j}+\alpha_{j} \delta_{j}^{-2} \chi_{j}\right)\right) u_{j} \geqslant \sum_{j \in \mathbb{Z}}\left(-\mu_{j}+\lambda\left(\alpha \nu_{j}+\alpha_{j} \delta_{j}^{-2} \chi_{j}\right)\right) \widehat{u}_{j} .
$$

Так как $\alpha+\sum_{|j| \leqslant N} \alpha_{j}=1$, то легко убедиться, что выполняется условие

$$
\left(\mathrm{b}_{4}\right) \alpha \sum_{j \in \mathbb{Z}} \nu_{j} \widehat{u}_{j}+\sum_{|j| \leqslant N} \alpha_{j} \delta_{j}^{-2} \widehat{u}_{j}=1
$$

Условия $\left(\mathrm{a}_{4}\right)$ и $\left(\mathrm{b}_{4}\right)$ достаточны для того, чтобы $\widehat{u}$ было решением задачи

$$
\sum_{j \in \mathbb{Z}} \mu_{j} u_{j} \rightarrow \max , \quad \alpha \sum_{j \in \mathbb{Z}} \nu_{j} u_{j}+\sum_{|j| \leqslant N} \alpha_{j} \delta_{j}^{-2} u_{j} \leqslant 1, \quad u_{j} \geqslant 0 .
$$


Следовательно, значения задач (17) и (18) совпадают.

Воспользуемся теперь леммой 2. Пусть $Y_{0}=l_{2}^{2 N+1} \times l^{\nu}$. Определим на $Y_{0}$ полускалярное произведение

$$
\left((x, z),\left(x^{\prime}, z^{\prime}\right)\right)_{Y_{0}}=\sum_{|j| \leqslant N} \alpha_{j} \delta_{j}^{-2} x_{j} \bar{x}_{j}^{\prime}+\alpha \sum_{j \in \mathbb{Z}} \nu_{j} z_{j} \bar{z}_{j}^{\prime}
$$

Определим оператор $I_{0}: X \rightarrow Y_{0}$ равенством

$$
I_{0} x(\cdot)=\left(\left\{x_{j}\right\}_{|j| \leqslant N}, F x(\cdot)\right)
$$

a $S: l_{\infty}^{2 N+1} \rightarrow Y_{0}-$ равенством $S y=(y, 0)$. Если $x(\cdot) \in W$ и $\left|x_{j}-y_{j}\right| \leqslant \delta_{j},|j| \leqslant N$, то

$$
\left\|I_{0} x(\cdot)-S y\right\|_{Y_{0}}^{2}=\alpha \sum_{j \in \mathbb{Z}} \nu_{j}\left|x_{j}\right|^{2}+\sum_{|j| \leqslant N} \alpha_{j} \delta_{j}^{-2}\left|x_{j}-y_{j}\right|^{2} \leqslant 1 .
$$

Согласно лемме 2 квадрат значения погрешности оптимального восстановления не превосходит значения задачи

$$
\|T x(\cdot)\|_{L_{2}(\mathbb{T})}^{2} \rightarrow \max , \quad\left\|I_{0} x(\cdot)\right\|_{Y_{0}}^{2} \leqslant 1
$$

которая после перехода к коэффициентам $Ф$ урье и замены $\left|x_{j}\right|^{2}$ на $u_{j}$ совпадает с задачей (18). Остается выписать оптимальный метод, который имеет вид $\widehat{\varphi}=T \circ \psi$, где коэффициенты $\Phi$ урье $\left\{\psi_{j}\right\}_{j \in \mathbb{Z}}$ функции $\psi=\psi(y)$ есть решение экстремальной задачи

$$
\sum_{|j| \leqslant N} \alpha_{j} \delta_{j}^{-2}\left|x_{j}-y_{j}\right|^{2}+\alpha \sum_{j \in \mathbb{Z}} \nu_{j}\left|x_{j}\right|^{2} \rightarrow \min , \quad x(\cdot) \in X .
$$

Легко видеть, что

$$
\psi_{j}= \begin{cases}y_{0}, & j=0 \\ \frac{\alpha_{j} \delta_{j}^{-2}}{\alpha \nu_{j}+\alpha_{j} \delta_{j}^{-2}} y_{j}, & 1 \leqslant|j| \leqslant p_{0}, \\ 0, & |j|>p_{0} .\end{cases}
$$

Проведя несложные вычисления, связанные с подстановкой выражений для $\alpha$ и $\alpha_{j}$, получаем требуемый результат.

\section{§4. Некоторые дальнейшие результаты}

4.1. Восстановление функций, заданных на сфере. Пусть

$$
\mathbb{S}^{d}=\left\{x=\left(x_{1}, \ldots, x_{d+1}\right) \in \mathbb{R}^{d+1}: \sum_{j=1}^{d+1} x_{j}^{2}=1\right\}
$$

- единичная $d$-мерная сфера. Известно (см. [9]), что $L_{2}\left(\mathbb{S}^{d}\right)=\sum_{k=0}^{\infty} H_{k}$, где $\operatorname{dim} H_{0}=n_{0}=1$,

$$
\operatorname{dim} H_{j}=n_{j}=\frac{2 j+d-1}{j}\left(\begin{array}{c}
j+d-2 \\
j-1
\end{array}\right), \quad j=1,2, \ldots
$$


$\left(H_{j}-\right.$ множество сфферических гармоник порядка $\left.j\right)$. Для оператора Лапласа $\Delta$ и любого $x(\cdot) \in H_{j}$ имеет место равенство

$$
\Delta x(\cdot)=-\Lambda_{j} x(\cdot)
$$

где $\Lambda_{j}=j(j+d-1)$. Пусть $\left\{Y_{k}^{j}\right\}_{k=1}^{n_{j}}$ - ортонормированный базис в $H_{j}$. Для $\beta>0$ оператор $(-\Delta)^{\beta / 2}$ определяется равенством

$$
(-\Delta)^{\beta / 2} x(\cdot)=\sum_{j \in \mathbb{N}} \Lambda_{j}^{\beta / 2} \sum_{k=1}^{n_{j}} x_{j k} Y_{k}^{j}(\cdot)
$$

где $x_{j k}=\left(x(\cdot), Y_{k}^{j}(\cdot)\right)_{L_{2}\left(\mathbb{S}^{d}\right)}-$ коэффициенты Фурье функции $x(\cdot)$.

Положим

$$
W_{2}^{\beta}\left(\mathbb{S}^{d}\right)=\left\{x(\cdot) \in L_{2}\left(\mathbb{S}^{d}\right):\left\|(-\Delta)^{\beta / 2} x(\cdot)\right\|_{L_{2}\left(\mathbb{S}^{d}\right)} \leqslant 1\right\} .
$$

Рассмотрим задачу оптимального восстановления оператора $T=(-\Delta)^{\gamma / 2}$ на классе $W_{2}^{\beta}\left(\mathbb{S}^{d}\right)$ по следуюшим информационным отображениям:

1) информация $I x(\cdot)=I_{\delta d} x(\cdot)$ о функции $x(\cdot) \in W_{2}^{\beta}\left(\mathbb{S}^{d}\right)$ задается в виде чисел $y_{j k}$ таких, что

$$
\sum_{j \in \mathbb{Z}_{+}} \sum_{k=1}^{n_{j}}\left|x_{j k}-y_{j k}\right|^{2} \leqslant \delta^{2}
$$

2) информация $I x(\cdot)=I_{\delta d}^{N_{m}} x(\cdot)$ о функции $x(\cdot) \in W_{2}^{\beta}\left(\mathbb{S}^{d}\right)$ задается в виде чисел $y_{j k}, j=0,1, \ldots, m, k=1, \ldots, n_{j}$, таких, что

$$
\sum_{j=0}^{m} \sum_{k=1}^{n_{j}}\left|x_{j k}-y_{j k}\right|^{2} \leqslant \delta^{2}
$$

(здесь $N_{m}=\sum_{j=0}^{m} n_{j}$ );

3) информация $I x(\cdot)=I_{\bar{\delta} d}^{N_{m}} x(\cdot)$ такова, что известны $y_{j k}, j=0,1, \ldots, m$, $k=1, \ldots, n_{j}$, такие, что

$$
\left|x_{j k}-y_{j k}\right| \leqslant \delta_{j k}, \quad j=0,1, \ldots, m, \quad k=1, \ldots, n_{j} .
$$

В работе [6] показано, что при всех $\lambda_{1}, \lambda_{2} \geqslant 0$ функция

$$
f(t)=-(t(t+d-1))^{\gamma}+\lambda_{1}+\lambda_{2}(t(t+d-1))^{\beta}
$$

может обращаться в нуль на множестве $[0,+\infty)$ не более чем в двух точках. Отсюда следует, что при $\beta>\gamma>0$ для $\mu_{j}=\Lambda_{j}^{\gamma}$ и $\nu_{j}=\Lambda_{j}^{\beta}$ выполнены условия 1)-3) из $\S 2$.

Применяя ту же схему доказательства, что и в теоремах 1-3, получаем их аналоги для рассматриваемых задач. Остановимся лишш на формулировках соответствующих результатов. В дальнейшем предполагаем, что всегда $\beta>\gamma>0$. 
ТЕОРема 4. Имеет место равенство

$$
E\left((-\Delta)^{\gamma / 2}, W_{2}^{\beta}\left(\mathbb{S}^{d}\right), I_{\delta d}\right)=\sqrt{\delta^{2} \Lambda_{s}^{\gamma}+\left(1-\delta^{2} \Lambda_{s}^{\beta}\right) \frac{\Lambda_{s+1}^{\gamma}-\Lambda_{s}^{\gamma}}{\Lambda_{s+1}^{\beta}-\Lambda_{s}^{\beta}}},
$$

ecлu $\Lambda_{s+1}^{-\beta / 2} \leqslant \delta<\Lambda_{s}^{-\beta / 2}, s \geqslant 1, u$

$$
E\left((-\Delta)^{\gamma / 2}, W_{2}^{\beta}\left(\mathbb{S}^{d}\right), I_{\delta d}\right)=d^{(\gamma-\beta) / 2}
$$

если $\delta \geqslant \Lambda_{1}^{-\beta / 2}$. При әтом метод

$$
\widehat{\varphi}(y)(\cdot)=\sum_{j \in \mathbb{N}} \Lambda_{j}^{\gamma / 2}\left(1+\Lambda_{j}^{\beta} \frac{\Lambda_{s+1}^{\gamma}-\Lambda_{s}^{\gamma}}{\Lambda_{s}^{\gamma} \Lambda_{s+1}^{\beta}-\Lambda_{s+1}^{\gamma} \Lambda_{s}^{\beta}}\right)^{-1} \sum_{k=1}^{n_{j}} y_{j k} Y_{k}^{j}(\cdot)
$$

является оптимальным, если $\Lambda_{s+1}^{-\beta / 2} \leqslant \delta<\Lambda_{s}^{-\beta / 2}, s \geqslant 1$, аесли $\delta \geqslant \Lambda_{1}^{-\beta / 2}$, то $\widehat{\varphi}(y)(\cdot)=0$ - оптимальный метод.

Положим

$$
s_{0}=s_{0}(m)=\min \left\{s \in \mathbb{N}: \frac{\Lambda_{s+1}^{\gamma}-\Lambda_{s}^{\gamma}}{\Lambda_{s+1}^{\beta}-\Lambda_{s}^{\beta}} \leqslant \Lambda_{m+1}^{\gamma-\beta}\right\} .
$$

TEOPEMA 5. $\Pi p u \delta \geqslant \Lambda_{s_{0}}^{-\beta / 2}$

$$
E\left((-\Delta)^{\gamma / 2}, W_{2}^{\beta}\left(\mathbb{S}^{d}\right), I_{\delta d}^{N_{m}}\right)=E\left((-\Delta)^{\gamma / 2}, W_{2}^{\beta}\left(\mathbb{S}^{d}\right), I_{\delta d}\right)
$$

u метод

$$
\widehat{\varphi}(y)(\cdot)=\sum_{j=1}^{m} \Lambda_{j}^{\gamma / 2}\left(1+\Lambda_{j}^{\beta} \frac{\Lambda_{s+1}^{\gamma}-\Lambda_{s}^{\gamma}}{\Lambda_{s}^{\gamma} \Lambda_{s+1}^{\beta}-\Lambda_{s+1}^{\gamma} \Lambda_{s}^{\beta}}\right)^{-1} \sum_{k=1}^{n_{j}} y_{j k} Y_{k}^{j}(\cdot)
$$

является оптимальным, если $\Lambda_{s+1}^{-\beta / 2} \leqslant \delta<\Lambda_{s}^{-\beta / 2}, 1 \leqslant s \leqslant s_{0}-1$, аесли $\delta \geqslant \Lambda_{1}^{-\beta / 2}$, mо $\widehat{\varphi}(y)(\cdot)=0$ - оптимальный метод. При $0<\delta<\Lambda_{s_{0}}^{-\beta / 2}$

$$
E\left((-\Delta)^{\gamma / 2}, W_{2}^{\beta}\left(\mathbb{S}^{d}\right), I_{\delta d}^{N_{m}}\right)=\sqrt{\delta^{2} \Lambda_{s_{0}}^{\gamma}+\left(1-\delta^{2} \Lambda_{s_{0}}^{\beta}\right) \Lambda_{m+1}^{\gamma-\beta}}
$$

$u$

$$
\widehat{\varphi}(y)(\cdot)=\sum_{j=1}^{m} \Lambda_{j}^{\gamma / 2}\left(1+\Lambda_{j}^{\beta} \frac{\Lambda_{m+1}^{\gamma}}{\Lambda_{s_{0}}^{\gamma} \Lambda_{m+1}^{\beta}-\Lambda_{m+1}^{\gamma} \Lambda_{s_{0}}^{\beta}}\right)^{-1} \sum_{k=1}^{n_{j}} y_{j k} Y_{k}^{j}(\cdot)
$$

- оптимальный метод.

Положим

$$
p_{0}=p_{0}(\bar{\delta})=\max \left\{p \in \mathbb{Z}_{+}: 1-\sum_{j=0}^{p} \Lambda_{j}^{\beta} \sum_{k=1}^{n_{j}} \delta_{j k}^{2}>0,0 \leqslant p \leqslant m\right\} .
$$


ТеОРема 6. Имеет место равенство

$$
E\left((-\Delta)^{\gamma / 2}, W_{2}^{\beta}\left(\mathbb{S}^{d}\right), I_{\bar{\delta} d}^{N_{m}}\right)=\sqrt{\Lambda_{p_{0}+1}^{\gamma-\beta}+\sum_{j=1}^{p_{0}}\left(\Lambda_{j}^{\gamma}-\Lambda_{j}^{\beta} \Lambda_{p_{0}+1}^{\gamma-\beta}\right) \sum_{k=1}^{n_{j}} \delta_{j k}^{2}}
$$

при этом метод

$$
\widehat{\varphi}(y)(\cdot)=\sum_{j=1}^{p_{0}} \Lambda_{j}^{\gamma / 2}\left(1-\left(\frac{\Lambda_{p_{0}+1}}{\Lambda_{j}}\right)^{\gamma-\beta}\right) \sum_{k=1}^{n_{j}} y_{j k} Y_{k}^{j}(\cdot)
$$

является оптимальным.

\section{2. Классы Харди-Соболева и Бергмана-Соболева на единичном} круге. Обозначим через $\mathscr{H}_{2}(D)$ пространство аналитических в единичном круге $D=\{z \in \mathbb{C}:|z|<1\}$ функций $x(\cdot)$, удовлетворяющих условию

$$
\|x(\cdot)\|_{\mathscr{H}_{2}(D)}=\sup _{0<\rho<1}\left(\frac{1}{2 \pi} \int_{\mathbb{T}}\left|x\left(\rho e^{i t}\right)\right|^{2} d t\right)^{1 / 2}<\infty
$$

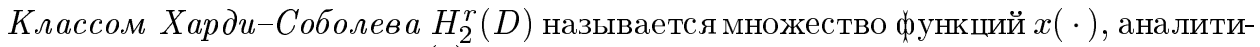
ческих в $D$, для которых $\left\|x^{(r)}(\cdot)\right\|_{\mathscr{H}_{2}(D)} \leqslant 1$.

Через $\mathscr{A}_{2}(D)$ обозначим пространство функций $x(\cdot)$, аналитических в единичном круге $D$ и удовлетворяющих условию

$$
\|x(\cdot)\|_{\mathscr{A}_{2}(D)}=\left(\int_{D}|x(z)|^{2} d \sigma(z)\right)^{1 / 2}<\infty
$$

где $\sigma$-плоская мера Лебега. Классом Бергмана-Соболева $A_{2}^{r}(D)$ называется множество функций $x(\cdot)$, аналитических в $D$, для которых $\left\|x^{(r)}(\cdot)\right\|_{\mathscr{A}_{2}(D)} \leqslant 1$.

Рассмотрим задачу оптимального восстановления $k$-й производной на классах $W=H_{2}^{r}$ и $A_{2}^{r}$ по информации о коэффициентах степенного ряда самой функции, заданных с погрешностью $\delta$ в норме пространства $l_{2}$. Иными словами, мы считаем, что для каждой функции $x(\cdot) \in W$ такой, что

$$
x(z)=\sum_{j \in \mathbb{Z}_{+}} a_{j} z^{j}
$$

известны числа $\left\{y_{j}\right\}_{j \in \mathbb{Z}_{+}}$такие, что

$$
\sum_{j \in \mathbb{Z}_{+}}\left|a_{j}-y_{j}\right|^{2} \leqslant \delta^{2}
$$

Соответствуюшее информационное отображение будем обозначать через $I_{\delta}^{+}$. Изучаемая задача оптимального восстановления записывается в виде

$$
E\left(D^{k}, W, I_{\delta}^{+}\right)=\inf _{\varphi: l_{2} \rightarrow \mathscr{W}} \sup _{\substack{x(\cdot) \in W, y \in l_{2} \\\left\|F^{+} x(\cdot)-y\right\|_{l_{2}} \leqslant \delta}}\left\|x^{(k)}(\cdot)-\varphi(y)(\cdot)\right\|_{\mathscr{W}}
$$


где $\mathscr{W}=\mathscr{H}_{2}(D), \mathscr{A}_{2}(D)$, a $F^{+} x(\cdot)=\left\{a_{j}\right\}_{j \in \mathbb{Z}_{+}}-$коэффициенты степенного ряда функции $x(\cdot)$.

Сформулируем аналог теоремы 1 , который может быть получен с помощью описанной выше схемы. Положим при фиксированных $k$ и $r(1 \leqslant k<r)$

$$
\begin{aligned}
& \mu_{j}(W)= \begin{cases}\left(\frac{j !}{(j-k) !}\right)^{2}, & j \geqslant k, W=H_{2}^{r}(D), \\
\left(\frac{j !}{(j-k) !}\right)^{2} \frac{1}{j-k+1}, & j \geqslant k, W=A_{2}^{r}(D), \\
0, & j<k,\end{cases} \\
& \nu_{j}(W)= \begin{cases}\left(\frac{j !}{(j-r) !}\right)^{2}, & j \geqslant r, W=H_{2}^{r}(D), \\
\left(\frac{j !}{(j-r) !}\right)^{2} \frac{1}{j-r+1}, & j \geqslant r, W=A_{2}^{r}(D), \\
0, & j<r .\end{cases}
\end{aligned}
$$

ТЕОРемА 7. При $W=H_{2}^{r}$ или $A_{2}^{r}$ и всех $1 \leqslant k<r$ для $\delta \geqslant \nu_{r}^{-1 / 2}(W)$ имеет место равенство

$$
E\left(D^{k}, W, I_{\delta}^{+}\right)=\sqrt{\delta^{2} \mu_{r-1}(W)+\frac{\mu_{r}(W)-\mu_{r-1}(W)}{\nu_{r}(W)}}
$$

а для $\nu_{s+1}^{-1 / 2}(W) \leqslant \delta<\nu_{s}^{-1 / 2}(W), s \geqslant r,-$ равенство

$$
E\left(D^{k}, W, I_{\delta}^{+}\right)=\sqrt{\delta^{2} \mu_{s}(W)+\left(1-\delta^{2} \nu_{s}(W)\right) \frac{\mu_{s+1}(W)-\mu_{s}(W)}{\nu_{s+1}(W)-\nu_{s}(W)}}
$$

При этом метод

$$
\widehat{\varphi}(y)(z)=\sum_{j=k}^{\infty}\left(1+\nu_{j}(W) \frac{\mu_{s+1}(W)-\mu_{s}(W)}{\mu_{s}(W) \nu_{s+1}(W)-\mu_{s+1}(W) \nu_{s}(W)}\right)^{-1} y_{j} z^{j-k}
$$

является оптимальным, если $\nu_{s+1}^{-1 / 2}(W) \leqslant \delta<\nu_{s}^{-1 / 2}(W), s \geqslant r$, а если $\delta \geqslant \nu_{r}^{-1 / 2}(W)$, то $\widehat{\varphi}(y)(z) \equiv 0$ - оптимальный метод.

Случаи, когда известно лишш конечное число коэффициентов степенного ряда с погрешностью в среднеквадратичной или равномерной метрике, могут быть рассмотрены точно так же, как это делалось для периодического случая. 


\section{Список литературы}

1. Micchelli C.A., Rivlin T.J. A survey of optimal recovery // Optimal estimation in approximation theory / ed. C.A. Micchelli, T.J. Rivlin. New York: Plenum Press, 1977. P. 1-54.

2. Melkman A.A., Micchelli C. A. Optimal estimation of linear operators in Hilbert spaces from inaccurate data // SIAM J. Numer. Anal. 1979. V. 16. P. 87-105.

3. Micchelli C.A., Rivlin T.J. Lectures on optimal recovery // Numerical analysis, Proc. SERC Summer Sch., Lancaster/Engl., 1984. Berlin: Springer-Verlag, 1984. P. 21-93. (Lecture Notes in Math. V. 1129.)

4. Micchelli C.A. Optimal estimation of linear operators in Hilbert spaces from inaccurate data: a second look // Numer. Algorithms. 1993. V. 5. P. 375-390.

5. Plaskota L. Noisy information and computational complexity. Cambridge: Cambridge Univ. Press, 1996

6. Магарил-Ильяев Г. Г., Тихомиров В. М. О неравенствах для производных колмогоровского типа // Матем. сб. 1997. Т. 188. № 12. С. 73-106.

7. Магарил-Ильяев Г. Г., Тихомиров В. М. Выпуклый анализ и его приложения. М.: Эдиториал УРСС, 2000.

8. Магарил-Ильяев Г. Г., Осипенко К. Ю., Тихомиров В. М. Оптимальное восстановление и теория экстремума // Докл. РАН. 2001. Т. 379. № 2. С. 161-164.

9. Стейн И., Вейс Г. Введение в гармонический анализ на евклидовых пространствах. М.: Мир, 1974.

Московский государственнњй институт радиотехники, электроники и автоматики (технический университет);

МАТИ - Российский государственный технологический

Поступила в редакцию университет им. К. Э. Циолковского

01.11.2001 